SHORT REPORT

\title{
A case of gliosarcoma appearing as ischaemic stroke
}

\author{
S Züchner, W Kawohl, B Sellhaus, M Mull, L Mayfrank, C M Kosinski
}

J Neurol Neurosurg Psychiatry 2003;74:364-366

\begin{abstract}
Objectives: Ischaemic stroke attributable to malignant brain tumour is a rarely reported phenomenon and even various imaging techniques including angiography do not necessarily lead to an accurate diagnosis.

Case description: A 46 year old, previously healthy man developed apoplectic symptoms with slight right sided hemiparesis and global aphasia. The computed tomography (CT) scan showed lesions of the left temporal lobe and the paraventricular white matter suggestive of left middle cerebral artery (MCA) infarction. Carotid angiography demonstrated compression of the MI segment of the MCA and occlusion of temporal MCA. The patient initially refused magnetic resonance imaging (MRI) because of claustrophobia. Because of fluctuating symptoms and successive worsening of the condition over weeks an MRI scan was conducted under general anaesthesia. Beneath temporal, opercular, and subcortical infarctions it revealed a left temporal tumour. A tumour biopsy disclosed a gliosarcoma (WHO grade IV). Microscopical examination of the surgical specimen demonstrated invasion of tumour cells into the wall of a greater pre-existing blood vessel.

Conclusions: Malignant brain tumours may cause ischaemic infarction. This is a rare but important differential diagnosis for the origin of strokes. The authors describe the first case with infiltration of intracranial blood vessels by tumour cells of a gliosarcoma.
\end{abstract}

schaemic strokes are most commonly caused by thrombotic obstructions of extracranial or intracranial vessels on the basis of atherosclerotic or non-atherosclerotic vasculopathies and "cardiac" or vascular cerebral embolism. Acquired or genetically determined coagulopathies and haematological diseases are rare causes. In contrast, cerebral neoplasms are known to induce apoplectic onset of neurological symptoms by intracranial haemorrhage. ${ }^{12}$ In very rare cases malignant tumours have provoke a sudden onset of neurological symptoms attributable to ischaemic stroke. ${ }^{3}$ Most commonly meningeomas have been known to cause stenosis or occlusion of intracranial vessels by vascular compression or encasement. ${ }^{45}$ Aoki et al describe a case of a malignant glioma causing a cerebral infarction by dissection of the middle cerebral artery caused by invasion of the vessel wall. ${ }^{6}$ Herman et al observed leptomeningeal dissemination of a malignant glioma, which mimicked cerebral vasculitis. ${ }^{7}$ Here we report a case with cerebral infarction as the apoplectic clinical manifestation of a gliosarcoma with atypical angiographic and neuropathological features.

\section{CASE REPORT}

A 46 year old man without significant medical history was admitted with acute onset of a slight right sided weakness and global aphasia. The symptoms were fluctuating. The neurological examination disclosed no additional deficits. Computed tomography (CT) scans demonstrated lesions of the left temporal lobe and the paraventricular white matter, which were initially interpreted as middle cerebral artery (MCA) infarction (fig lA, 1B). Because of claustrophobia the patient refused magnetic resonance imaging (MRI). Doppler sonography of the extracranial and intracranial arteries revealed no stenosis, occlusion, or dissection. Transoesophageal echocardiography, 24 hour Holter monitoring, and blood screens for coagulopathies were completely normal. The symptoms subsided within 10 days to a mild mixed aphasia and the patient was dismissed with clopidogrel. Five days later the aphasia worsened again and the patient developed a right sided hemiparesis. A CT follow up scan disclosed a progressive lesion extending from the left temporal region to the left operculum and the left paraventricular white matter. Transcranial duplex sonography of the cerebral arteries now revealed narrowing of the trunk of the left MCA. Cerebral angiography showed narrowing of the sphenoidal, insular and opercular parts of the left MCA and occlusion of the left temporal arteries at the MCA bifurcation (fig 1C). No pathological vessels indicating a tumour were observed. Repeated cerebrospinal fluid examinations showed mild lymphocytic pleiocytosis (15 cells $/ \mu \mathrm{l}$ ) but no specific germ or tumour cells. Despite negative results of serological examinations an isolated vasculitis of the CNS seemed still possible and the patient received subsequently on a trial basis a treatment with corticosteroids leading to a temporary improvement of the condition over six weeks. Because of fluctuating symptoms and clinical deterioration MRI was conducted under general anaesthesia seven weeks after readmission. In addition to the known infarctions it showed a hyperintense temporal lesion with gadolinium enhancement, suggesting a neoplasm (fig lD). The patient underwent subtotal removal of the tumour via right frontotemporal craniotomy. The postoperative course was uncomplicated. Neuropathological examination revealed a malignant brain tumour presenting a biphasic tissue pattern with gliomatous and mesenchymal components. Gliomatous areas showed typical features of a glioblastoma with remarkable nuclear atypia, ischaemic necroses, increased mitotic index, and glial fibrillary acidic protein (GFAP) expression. The mesenchymal component consisted of areas with densely packed long bundles of spindle cells in a storiform pattern. Immunohistochemically the cells of this section expressed vimentin and were surrounded by a dense network of reticulin fibres as typical features of sarcomatous cells. Within the gliomatous section GFAP positive tumour cells infiltrated the whole adventitia and muscle layer up to the intima of one large blood vessel (fig 2). Whether the vessel is venous or arterial is difficult to determine because of the deep infiltration of the tumour cells into the wall. Although the vessel is related to a necrotic area, a complete occlusion was not seen. The tumour was classified as a gliosarcoma (WHO grade IV). Additionally, the patient received $60 \mathrm{~Gy}$ of radiation therapy. Eight months later he died. The relatives refused a postmortem examination.

Abbreviations: $M R I$, magnetic resonance imaging; $C T$, computed tomography; MCA, middle cerebral artery 

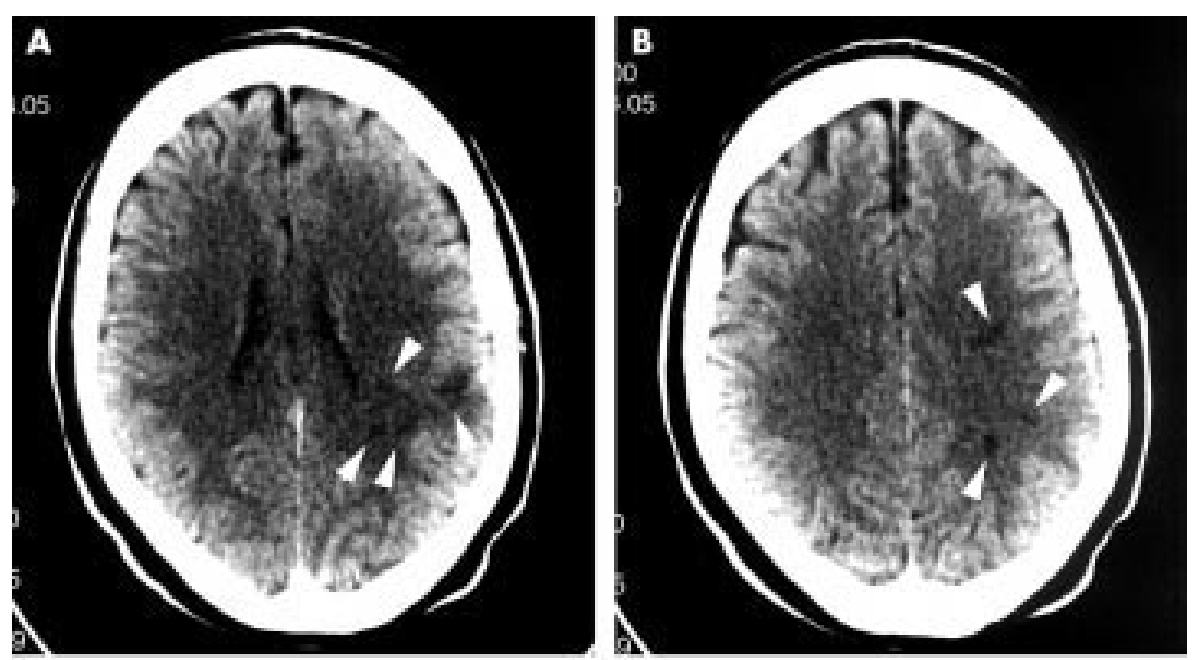

Figure 1 Neuroradiological findings. (A, B) Cranial CT scans two days after onset of symptoms show small hypodense lesions of the left centrum semiovale (arrows). (C)

Posteroanterior view of a left carotid arteriogram three weeks after onset of symptoms revealed stenosis and occlusion of the left temporal artery (arrow). (D) MRI scan six weeks after onset of symptoms reveals a hyperintense lesion of the left temporal lobe in the T1 weighted image with contrast enhancement (arrow) right sided developmental venous anomaly.
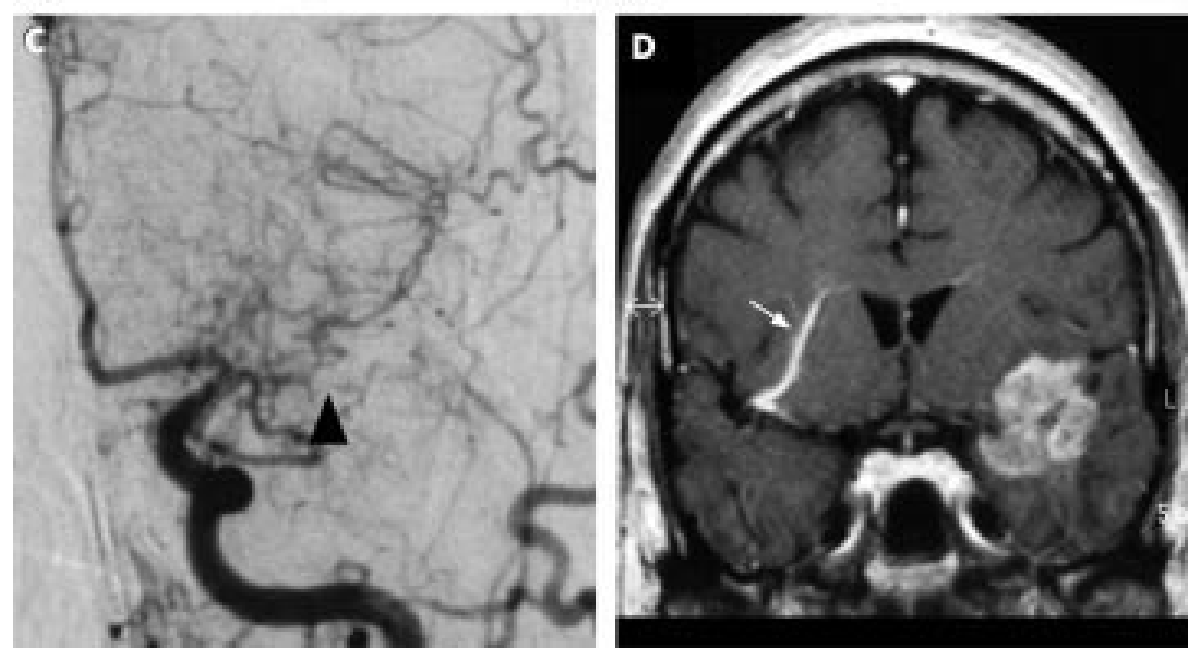

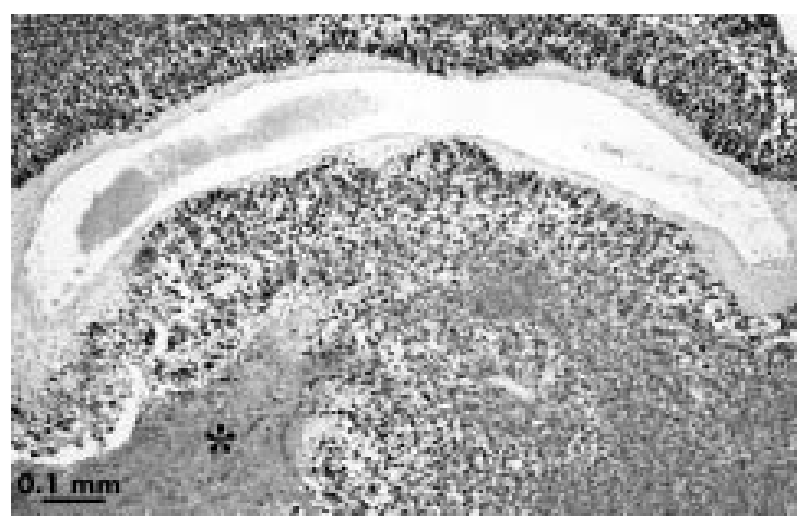

Figure 2 Tumour specimen immunohistochemically stained with antibody against GFAP. The large vessel (inner diameter $0.16 \mathrm{~mm}$ ) is infiltrated by GFAP positive gliomatous tumour cells, but not occluded. Pleomorphic tumour cells were apparent in the adventitia and media of the vessel wall, $\left({ }^{*}\right)$ necrotic area.

\section{DISCUSSION}

The sudden onset of clinical symptoms and the hypodense lesions in the CT scans ( fig lA, 1B) were initially considered as a "classic" ischaemic stroke. The recurrent infarctions and the mild lymphocytosis of the cerebrospinal fluid, the narrowing of MCA branches in the cerebral angiography (fig $1 C$ ) as well as the clinical stabilisation under corticosteroid treatment over six weeks were suggestive of a cerebral vasculitis. The lack of typical signs made it difficult to diagnose a malignant tumour. Moreover, the claustrophobia of the patient made it impossible to conduct MRI, initially.

It has occasionally been observed that gliomatous cells are able to invade the arterial wall and may induce weakening of the wall leading to arterial dilatation or rupture. ${ }^{89}$ However, gliosarcoma is an uncommon malignant brain tumour with mixed glial and mesenchymal elements. The neuropathological examination of the presented case demonstrated the invasion of the wall of a major blood vessel by partially necrotic gliomatous tumour cells ( fig 2 ). Tumours usually develop more irregular vessels. Thus, we argue that this vessel is not a tumour neovascularisation but a pre-existing blood vessel. The authors propose a haemodynamically induced low flow infarction attributable to compression and progressive occlusion of MCA branches by tumour cells. ${ }^{10}$ Although the intima of the observed vessel was intact in other sections a breach of the intima may induce vascular thrombosis and arterioarterial embolism. This is the first reported stroke resulting from invasion of the wall of intracranial arteries by gliomatous cells of a gliosarcoma. The reason why a small subgroup of malignant gliomas becomes primarily symptomatic by infiltrating the walls of the blood vessels instead of the extending volume remains to be explained.

In conclusion, tumour infiltration of major intracranial blood vessels by malignant gliosarcoma can cause ischaemic infarction. In early stages of the disease it can be a difficult but important differential diagnosis for recurrent cerebral infarction. 


\section{Authors' affiliations}

S Züchner, C M Kosinski, Department of Neurology, Aachen University Hospital, Aachen, Germany

S Züchner, B Sellhaus, Department of Neuropathology, Aachen University Hospital

W Kawohl, Department of Psychiatry, Aachen University Hospital

M Mull, Department of Neuroradiology, Aachen University Hospital

L Mayfrank, Department of Neurosurgery, Aachen University Hospital

Competing interests: none declared.

Correspondence to: Dr S Züchner, Department of Neuropathology,

University Hospital of Aachen, Pauwelsstrasse 30, 52074 Aachen,

Germany; stephan.zuechner@post.rwth-aachen.de

Received 5 June 2002

In revised form 23 September 2002

Accepted 23 October 2002

\section{REFERENCES}

1 Kondziolka D, Bernstein M, Resch L, et al. Significance of hemorrhage into brain tumours: clinicopathological study. J Neurosurg 1987:67:852-7.
2 McCormick WF, Rosenfield DB. Massive brain hemorrhage: a review of 144 cases and an examination of their causes. Stroke 1973;4:946-54.

3 Fisher $M$, Recht LD. Brain tumour presenting as an acute pure motor hemiparesis. Stroke 1989:20:288-91.

4 Launay M, Fredy D, Merland JJ, et al. Narrowing and occlusion of arteries by intracranial tumours. Review of the literature and report of 25 cases. Neuroradiology 1977;14:117-26.

5 Spallone A. Occlusion of the internal carotid artery by intracranial tumours. Surg Neurol 1981;1:51-7.

6 Aoki N, Sakai T, Oikawa A, et al. Dissection of the middle cerebral artery caused by invasion of malignant glioma presenting as acute onset of hemiplegia. Acta Neurochir 1999;141:1005-8.

7 Herman C, Kupsky WJ, Rogers L, et al. Leptomeningeal dissemination of malignant glioma simulating cerebral vasculitis. Case report with angiographic and pathological studies. Stroke 1995;26:2366-70.

8 Hart MN, Byer JA. Rupture of middle cerebral artery branches by invasive astrocytoma. Neurology 1974;24:1171-4.

9 Cowen RL, Siqueira EB, George E. Angiographic demonstration of a glioma involving the wall of the anterior cerebral artery. Report of a case. Radiology 1970;97:577-8.

10 Mull M, Schwarz M, Thron A. Cerebral hemispheric low-flow infarcts in arterial occlusive disease. Lesion patterns and angiomorphological conditions. Stroke 1997;28:118-23.

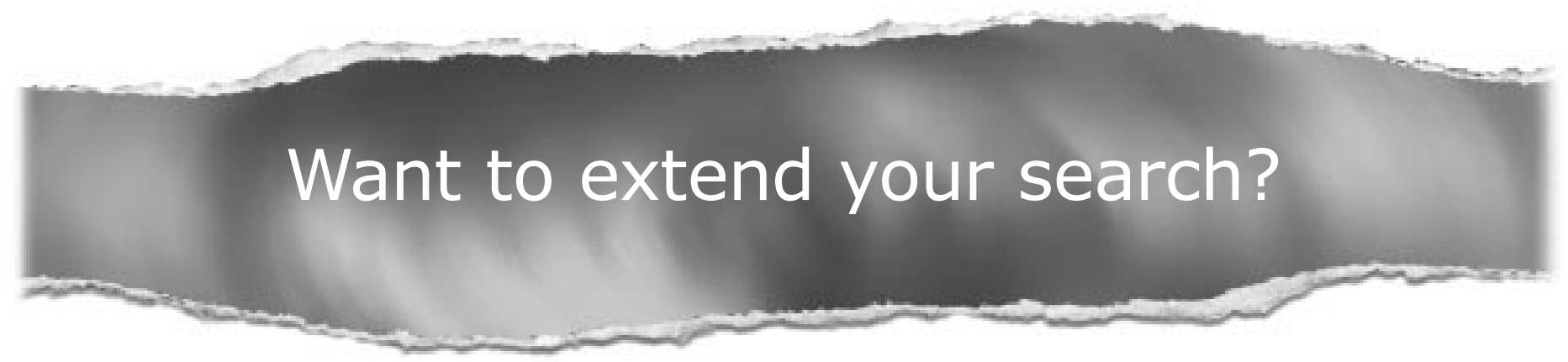

\section{Cross journal searching}

If you can't find what you are looking for in the Journal of Neurology, Neurosurgery, and Psychiatry you can extend your search across many of the more than 200 journals available for selection. You can restrict your search to specific subject areas (eg, clinical medicine, basic research), or select specific journals, or search all available titles.

\section{www.jnnp.com}

